\title{
The Effect of Vestibular Stimulation Exercises on Balance, Coordination, and Agility in Children with Down Syndrome
}

\author{
Kathy Carter, Sarah Sunderman, Stefanie Wooten Burnett \\ Department of Health and Sport Science, University of Louisville, Louisville, USA \\ Email address: \\ Kathleen.carter@louisville.edu(K. Carter),ssunderman@sandalwood.com (S. Sunderman), \\ Swooten-burnett@gccschools.com(S.W. Burnett)

\section{To cite this article:} \\ Kathy Carter, Sarah Sunderman, Stefanie Wooten Burnett. The Effect of Vestibular Stimulation Exercises on Balance, Coordination, and \\ Agility in Children with Down Syndrome. American Journal of Psychiatry and Neuroscience. Vol. 6, No. 2, 2018, pp. $28-32$. \\ doi: 10.11648/j.ajpn.20180602.11
}

Received: April 30, 2018; Accepted: May 15, 2018; Published: May 31, 2018

\begin{abstract}
Background. Children with Down syndrome (DS) demonstrate vestibular, sensory, motor and perceptual impairments which manifests as decreased levels of balance, strength, and motor coordination. Together these issues may decrease functional ability leading to more sedentary lifestyles. Use of vestibular stimulation therapy has been attempted to assist in improving motor control and balance in this population. Objective. The objective of this study was to determine the effect of a vestibular stimulation exercise program on balance, coordination and agility in children with DS. Methods. Seventeen children with DS were recruited from two summer enrichment programs and were divided into two groups based on age (group 1: 9.9 yrs \pm 2.8 ; group 2: 18.4 yrs. \pm 1.7 ). Assessments were completed using BOT2 subtests for balance, bilateral and upper limb coordination, and agility prior to and after six weeks of twice weekly vestibular stimulation exercises. Results. Both groups showed improvement in upper limb coordination and agility, while group 2 demonstrated improvement in one of the balance subtests. Conclusion. These results suggest a vestibular stimulation exercise program could increase balance and agility in children with DS and possibly assist in increasing their functional ability.
\end{abstract}

Keywords: Down Syndrome, Vestibular Stimulation, Balance, Agility, BOT2

\section{Introduction}

Down syndrome (DS) is a genetic disorder which includes a full or partial copy of chromosome 21 [1]. It occurs with equal frequency in people of different nationalities, social backgrounds, and economic classes, averaging 1 in about every 600 births worldwide $[1,2]$. Children with DS have a number of motor characteristics such as hypotonicity, joint hypermobility, decrease in deep tendon reflexes, and a delay in developing reaction timing and equilibrium reactions, which could cause a delay in achieving developmental stages [3-5]. These children often demonstrate decreased levels of balance and motor coordination, as well as sensory integration and vestibular deficits [6].

The vestibular system plays a major role in early motor behavior [5, 7] with the primary functions of maintaining balance, posture and equilibrium by monitoring motion of the head, and stabilizing the eyes relative to the environment [8]. Vestibular malformations are common in children with DS
$[9,10]$. Individuals with vestibular disorders have difficulty maintaining equilibrium leading to decreased postural control and poor balance while making it difficult to select an accurate source of sensory information leading to sensory integration dysfunction.

Sensory integration is the ability of the central nervous system to gather information such as movement, touch, vision and hearing, put them together with prior information, memories, and knowledge to make an appropriate response to one's environment. Sensory integration dysfunction creates difficulties with balance and positional awareness. Vestibular malformations may cause children with DS have sensory integration dysfunction [5] which could decrease their functional abilities.

When sensory integration and vestibular deficits exist, clinicians have attempted to facilitate normal motor development through a variety of techniques including sensory integrative therapy and vestibular stimulation therapy. Vestibular rehabilitation, which encompasses both 
sensory integrative and vestibular stimulation therapies, refers to utilizing exercises such as repositioning techniques, and exercises to improve muscle force and balance [11]. Studies have shown that stimulating the vestibular system of individuals who have vestibular dysfunction have resulted in improved motor control and balance $[5,7]$.

Minds-in-Motion (MiM) is a developmental program with the goal of improving children's visual processing and motor skills $[12,13]$. The premise of MiM is that there is a link between early afferent neural stimulation and cognitive abilities [14]. Specifically, movement activities that stimulate the vestibular system can have an impact on children's academic and physical domains [14]. Although implemented in several schools, MiM has never been investigated in the DS population. Therefore, the purpose of this study was to determine the effect of the MiM vestibular stimulation exercise program on balance, upper limb coordination, bilateral coordination, and agility in children with DS.

\section{Methods}

\subsection{Participants}

A convenience sample was recruited from two summer enrichment programs designed specifically for individuals with DS. The first program was specific for children 5-14 years of age while the second program encompassed ages 1520 years. Nine children (6 male, 4 female) participated in the younger age group (group 1) and eight children (4 male, 4 female) participated from the older age group (group 2). Inclusion criteria were 1) ability to walk independently, 2) ability to follow simple directions, and 3) attendance at the summer program on days and times when the intervention occurred. Parents/guardians attended an informational meeting and then signed informed consents and children signed assent forms. The University Institutional Review Board approved this study.

\subsection{Testing}

Physical testing took place in areas away from the main program activities to minimize distraction. Physical assessments for group 1 took place in a recreation room, which had a large open area at the front. Group 2 assessments took place in a gymnasium.

The primary investigator escorted all participants to the testing area to assist in establishing a level of comfort with participants. All physical assessments conducted were subtests within the Bruininks Oseretsky Test of Motor Proficiency (BOT2) [15] which has been shown to be a reliable and valid measure of fine and gross motor skills and motor proficiency in the DS population $[16,17,18]$. The subtests utilized were balance, bilateral coordination, upperlimb coordination, and agility. Items in the balance subtest were walking forward on a line and standing on one leg (eyes open and eyes closed). Item for bilateral coordination was touching nose with index fingers-eyes closed. Upper limb coordination subtest utilized dropping and catching a ball and catching a tossed ball.
Stepping sideways over a balance beam was assessed agility. The primary investigator conducted all assessments, in the same order for each participant. Participants completed two trials for each test with the highest score being utilized for data analysis [15].

\subsection{Intervention}

An exercise circuit, or maze, was developed to provide vestibular stimulation exercises in a fun atmosphere [14]. The maze approach included 15 activities that involved skillrelated components such as balance and coordination as well as health-related components such as muscular strength and endurance, flexibility, and cardiovascular endurance [13]. Each station was set up in the same order, in the same place each day and participants performed one set at each station per session. Two 10 minute sessions were completed twice per week for 6 weeks. Participants went through the stations individually to minimize distractions. Exercises included in the maze per MiM protocol [14] were as follows:

Strong Arm Push: Participants stood facing a wall, one foot ahead of the other, arms stretched forward with palms pressed flat on the wall at shoulder height. Participants pushed the wall with as much force as possible for 10 seconds. The verbal cue was "push the wall down".

Eye Can Convergence: A 5 feet length of string was held at the children's eye level, between the investigator and the participant, parallel to the floor. Three $1 \frac{1}{2}$ inch foam balls of varying colors were attached evenly spaced along the string. While holding the string participants cue was to "focus on each ball one at a time for a count of ten".

Eye to Eye: Participants stood 14 inches in front of the investigator and followed the top of a pencil with their eyes, without moving their head. The pencil was moved in two horizontal motions, two vertical motions, two circles clockwise, two circles counterclockwise, two horizontal movements, and ended with the pencil moving towards the participant's nose twice.

The Beam Team: Participants walked along a $2 \frac{1}{2}$ in. X 5 in. X $10 \mathrm{ft}$. foam balance beam with arms extended out to the side. Participants cue was to focus on a specific point in front of them.

Jelly Roll: Participants lay on a $4 \mathrm{ft}$. x $8 \mathrm{ft}$. tumbling mat with legs straight and arms stretched above the head, then rolled the length of the mat keeping the body straight.

Puppy Dog Crawl: Participants kneeled on a $4 \mathrm{ft}$. x $8 \mathrm{ft}$. tumbling mat with palms flat on the floor. The instruction was to crawl across the mat, moving the opposite arm with the opposite leg.

Monster Mash: Six 9 in. circular pads were placed on the floor in a straight line touching each other. Participants stomped one foot at a time with as much force as possible on each circle.

Climb Every Mountain: Participants stepped over 3 plastic hurdles set at heights of 6 in., 8 in., and 10 in. leading with their preferred foot and placing both feet on the ground before starting the next hurdle.

Balance Board Bash: Participants placed their feet equal 
distance from the center of a balance board and maintained the board in a horizontal position as long as possible.

Electric Slide: Participants stood with their body facing, but not touching, a wall. Without turning their head or body the participants moved sideways along the length of the wall by taking a side step with the lead foot, sliding the following foot along until it touches the lead foot.

Skip to My Lou: Participants skipped across the room, swinging their arms laterally across the body in an exaggerated fashion raising knees as high as possible.

Cross Walk: Participants walked across the room lifting knees high while touching alternating knees with opposite elbows.

Bean Bag Boogie: Participants tossed a bean bag in the air and caught it while walking in a straight line across the room. Participants were cued to "follow the bean bag with your eyes".

Jumping Jack Flash: Participants stood behind a line and jumped over a second line placed $3 \mathrm{ft}$. away, taking off and landing on both feet.

Step Back: Participants walked backwards up 3 stairs, then forward down 3 stairs, then backwards up 3 stairs looking straight ahead. The investigator was positioned directly in front of participants for safety.

\section{Results}

Nine individuals from group 1 (5-14 yrs.) and 8 individuals from group 2 (15-20 yrs.) participated in the study. (Table 1). Twelve sessions were available to group 1 with $100 \%$ attendance. Group 2 had a total of 11 sessions available after one session was canceled due to a schedule conflict. Group attendance was $97.7 \%$, with 1 participant missing 2 sessions due to a family vacation

Table 1. Demographic information by age group

\begin{tabular}{lll}
\hline Characteristic & Group 1 (n=10) & Group 2 $(\mathbf{n}=\mathbf{9})$ \\
\hline Gender & $\mathrm{M}=6, \mathrm{~F}=4$ & $\mathrm{M}=4, \mathrm{~F}=4$ \\
Age $($ years $)$ & $9.9(5-14)$ & $18.4(15-20)$ \\
Weight $(\mathrm{kg})$ & $34.8(24.1-49.6)$ & $67.4(45.3-110.5)$ \\
Height $(\mathrm{cm})$ & $133.4(101.6-162.6)$ & $159.1(142.2-165.1)$ \\
\hline
\end{tabular}

Statistical analysis was completed on SPSS v.21.0 (IBM, Inc., Chicago, IL) using a within subjects paired samples ttest to determine differences in pre- and post-test scores.

\subsection{Group 1}

Table 2 lists results and statistical analysis. There was no significant change in overall balance. Individual tests within the balance category were all non-significant.

Bilateral coordination did not achieve statistical significance.

Overall upper limb coordination improved significantly. Catching the Ball with Both Hands, showed significant improvement while, Catching a Dropped Ball, did not.

Agility showed significant improvement.

Table 2. Group 1 pre-test and post-test scores for BOT2 subtests in balance, bilateral coordination, upper limb coordination and speed and agility.

\begin{tabular}{lllll}
\hline Test & Pre (pts) & Post (pts) & p-value & Effect size (r) \\
\hline Balance & 5.78 & 6.78 & 0.282 & 0.378 \\
WFL & 2.67 & 3.11 & 0.347 & 0.111 \\
SLS-EO & 1.11 & 1.44 & 0.397 & 0.719 \\
SL-EC & 2.00 & 2.22 & 0.130 \\
Bilateral Coordination & & & 0.347 \\
TN-EC & 2.89 & 3.00 & $0.024 *$ & 0.111 \\
Upper Limb Coord. & 3.56 & 6.56 & $0.017 *$ & 0.701 \\
CTB-BH & 1.89 & 3.22 & 0.319 \\
CDB-BH & 1.67 & 3.33 & 0.620 \\
Agility & & & $0.001 *$ \\
SSBB & 3.78 & 5.00 & 0.889 \\
\hline
\end{tabular}

*Denotes statistical significance.

\subsection{Group 2}

Table 3 lists results and statistical. Overall balance revealed no significant changes. There were no significant differences for the tests within the balance category for Standing on One Leg - Eyes Open or Standing with Feet Apart on a Line- Eyes Closed; however, the test Walking Forward on a Line showed statistically significant improvement.

Bi-lateral coordination revealed no significant changes.

The upper limb coordination showed significant improvement. Catching a Tossed Ball with Both Hands showed significant improvement while Catching a Dropped Ball did not.

Agility test showed significant improvement.

Table 3. Group 2 pre-test and post-test scores for BOT2 subtests in balance, bilateral coordination, upper limb coordination and speed and agility.

\begin{tabular}{lllll}
\hline Test & Pre (pts) & Post (pts) & p-value & Effect size (r) \\
\hline Balance & 5.50 & 7.50 & 0.180 & 0.756 \\
WFL & 2.25 & 3.25 & $0.007 *$ & 0.817 \\
SLS-EO & 1.63 & 1.75 & 0.598 & 0.204 \\
SL-EC & 1.63 & 2.50 & 0.111 & 0.568 \\
Bilateral Coordination & & & & \\
\hline
\end{tabular}




\begin{tabular}{lllll}
\hline Test & Pre (pts) & Post (pts) & p-value & Effect size (r) \\
\hline TN-EC & 2.25 & 2.75 & 0.104 & 0.577 \\
Upper Limb Coord. & 4.75 & 6.25 & $0.009^{*}$ & 0.802 \\
CTB-BH & 2.50 & 3.50 & $0.007^{*}$ & 0.817 \\
CDB-BH & 2.25 & 2.75 & 0.170 & 0.511 \\
Agility & & & & 0.904 \\
SSBB & 3.25 & 5.00 & $0.001^{*}$ & \\
\hline
\end{tabular}

*Denotes statistical significance.

\section{Discussion}

This study determined the effect of a vestibular stimulation exercise program on balance, upper limb coordination, bilateral coordination, and agility on children with DS. Results of this study indicate vestibular stimulation exercises can have a positive effect on balance, upper limb coordination and agility in both age groups, and balance in older children.

Studies of vestibular stimulation on individuals with Down syndrome are few. Early studies [19, 20] determined vestibular stimulation training had a positive effect on motor skills of children with DS. More recently, 3 groups of children with DS received either sensory integrative therapy, vestibular stimulation in addition to sensory integrative therapy, or neurodevelopmental therapy [5]. Results indicated the vestibular stimulation group improved in fine motor skills, reflex development and gravitational insecurity [5]. The results of the current study are in agreement with the previous study in that reflex development and gravitational insecurity align with agility and balance.

While overall balance did not improve in either group. Two measures of static balance, single leg stance in eyes open and eyes closed positions, also did not change. Individuals with DS tend to have greater sway while in static positions and it was felt the current study period was not long enough to elicit a change in sway. Walking on a Line is an indicator of dynamic balance, which can be improved by changing adaptation strategies. The older participants may have been able to create adaptations, which allowed greater dynamic balance. In a previous study it was hypothesized the lack of overall balance improvement was due to the median score before high and leaving little room for improvement [17]. In this study the scores for all three balance subtests were very close to the maximum attainable indicating the ceiling effect may have been an issue.

Bi-lateral coordination is when both sides of the body work together in a coordinated fashion and is utilized in basic motor skills, such as walking and reaching to pick up objects. This study indicated no change in bi-lateral coordination. This may be due to only one measurement, which required participants to have their eyes closed. No maze exercises were performed with eyes closed so there was no specificity of training. This is in agreement with Wang, Chiang, Su and Wang [21] who did not find changes in bi-lateral coordination when the Wii was utilized as a treatment modality. Several exercises in the maze required visual-spatial skills; knowing the position of objects in space, which could improve bilateral coordination. It may be that poor motor planning activities are limiting this parameter and may require serial activities, such as nesting cups, in order to improve.

Upper limb coordination is necessary to coordinate arm and hand movements with visual tracking. Few studies have looked specifically at upper limb coordination. One study saw significant change in upper limb coordination after use of a Wii 3 times per week for 8 weeks [21]. In another study, motor outcomes in a child with DS after playing a Wii also found upper limb coordination was improved [23]. This was attributed to the precision of arm movements and timing required during Wii games. The exercise "bean bag boogie" used the same movements as the assessment "Catching a tossed ball - both hands". The use of comparable motions provided more practice and may be indicative of a need for increased numbers of repetitions to improve motor skills in children with DS.

Agility is the ability to change directions efficiently and requires the integration of motor skills such as balance, strength, and reaction time. It is indicative of an individuals' ability to react to their environment. Both groups showed significant improvement in agility. This is in agreement with study that saw significant improvement in agility in children with DS following a 6 week intervention of 20 minutes of exercise through use of a Wii with [24]. Wuang et al., (2011) [20] showed improved agility performance in children with intellectual disabilities after 6 months of sensorimotor training.

Small sample size may have decreased our ability to detect small, but consistent changes in some parameters. The intervention was provided for the entire 6 week programs. Other studies have shown improvement in motor skills in six weeks with interventions provided a minimum of 3 times per week [5, 17] or if interventions were administered twice per week for longer time periods [24]. A similar study conducted during a school year in a physical education class could possibly allow for a larger sample size as well as a control group.

Vestibular stimulations has been successfully used to improve physical functioning of children with myelomeningocele [25], Williams syndrome [26], and cerebral palsy [27]. The possibility of vestibular stimulation therapy improving motor functioning of children with DS should be explored further in studies with larger numbers of participants with longer intervention times.

\section{Discussion}

The results of this study are in agreement with the small number of previous studies utilizing various methods of vestibular stimulation to improve motor functioning of children with DS. Children with DS tend to lack motivation 
and may lose attention easily. The MiM maze exercises are fun and the variety assists in keeping participants attention focused on their body movement. This "fun" element may be a key to increasing motivation during activities and maximizing the therapeutic effect of the program.

\section{References}

[1] National down Syndrome Society. www.ndsr.org/about-downsyndrome/down-syndrome-facts/. retrieved June 2017.

[2] Nadkarni, D. (2012). Enhancing eye-hand coordination with therapy intervention to improve visual-spatial abilities using 'The retraining approach' in children with Down syndrome: Three case studies. Disability, CBR \& Inclusive Development Journal, 23 (2), 107-120.

[3] El-Maksoud, G., Abd-Elmonen, A., \& Rezk-Allah, S. (2016) Effect of individual and group sensory-perceptual motor training on motor proficiency and quality of life in children with Down syndrome. International Journal of Therapies and Rehabilitation Research, S (4), 37-45.

[4] Sacks, B., \& Buckley, S. (2003). What do we know about the movement abilities of children with Down syndrome? Down Syndrome News and Update, 2(4), 131-141.

[5] Uyanik, M., Bumin, G., \& Kayihan, H. (2003). Comparison of different therapy approaches in children with Down syndrome. Pediatrics International, 45, 68-73.

[6] Costa, A. C. (2011). An assessment of the vestibulo-ocular reflex (VOR) in persons with Down syndrome. Experimental Brain Research, 214(2), 199-213. doi:10. 1007/s00221-0112820-y.

[7] Maher, K. (2015). Vestibular stimulation systems and methods of use. US9183756 B2.

[8] Menear, K. (2007). Parents' perceptions of health and physical activity needs of children with Down syndrome. Down Syndrome Research and Practice, 12(1), 60-68.

[9] Blaser, S., Propst, E., Martin, D., Feigenbaum, A., James, A., Shannon, P., \& Popsin, B. (2006). Inner ear dysplasia is common in children with Down syndrome (trisomy 21). Largyngoscope, 116, 2113-2119.

[10] Han, F., Yu, H., Zhang, J., Tian, C., Schmidt, C., Nava, C., Davisson, M., \& Zheng, L. (2009). Otitis media in a mouse model for Down syndrome. International Journal of Experimental Pathology, 90, 480-488.

[11] Schubert, M. C., \& Minor, L. B. (2004). Vestibulo-ocular physiology underlying vestibular hypofunction. Physical Therapy, 84(4), 373-385.

[12] Meyer, C. (2012). Minds-in-Motion, the Maze Handbook. Minds-in-Motions, Inc. Press.

[13] Vidoni, C., Lorenz, D., \& Terson de Paleville, D. (2014). Incorporating a movement skill program into a preschool daily schedule. Early Childhood Development \& Care Journal, 184(8), 1211-1222.

[14] Bruinincks, R. H., \& Bruininks, B. D. (2005). Test of motor proficiency. Manual (2nd ed.). Minneapolis, MN: Pearson Assessments.
[15] Connolly, B. H., Morgan, S. B., Russell, F. F., \& Fullinton, W. L. (1993). A longitudinal study of children with Down syndrome who experienced early intervention programming. Physical Therapy, 73(3), 170-179; discussion 179-181.

[16] Gupta, S., Rao, B. K., \& Kumaran, S. (2011). Effect of strength and balance training in children with Down's syndrome: a randomized controlled trial. Clinical Rehabilitation, 25(5), 425-432. doi: $10.1177 / 0269215510382929$

[17] Wuang, W. Y., \& Ju, Y. H. (2002). Promoting balance and jumping skills in children with Down syndrome. Perceptual Motor Skills, 94(2), 443-448. doi:10. 2466/pms. 2002. 94. 2. 443.

[18] Kantner, R., Clark, D., Allen L., \& Chase, M. (1976). Effects of vestibular stimulation on nystagmus response and motor performance in the developmentally delayed infant. Physical Therapy, 56, 414-421.

[19] Mclean, W., \& Baumeister, A. (1982). Effects of vestibular stimulation on motor development and stereotyped behavior of developmentally delayed children. Journal of Abnormal Child Psychology, 10, 229-245.

[20] Wuang, Y., Chiang, C., Su, C., \& Wang, C. (2011). Effectiveness of virutal reality using Wii gaming technology in children with Down syndrome. Research in Developmental Disabilities, 23, 312-321.

[21] Silva, V., Campos, C., Sa, A., Cavadas, M., Pinto, J., Simoes, P., Machado, S., Murillo-Rodriguez, E., \& Barbosa-Rocha, N. (2017). Wii-based exercise program to improve physical fitness, motor proficiency and functional movility in adults with Down syndrome. Journal of Intellectual Disability Research, 61(9), 755-765.

[22] Berg, P., Becker, T., Martian, A., Primrose, K. D., \& Wingen, J. (2012). Motor control outcomes following Nintendo Wii use by a child with Down syndrome. Pediatric Physical Therapy, 24(1), 78-84. doi:10. 1097/PEP. 0b013e31823e05e6

[23] Lin, H., \& Wuang, Y. (2012). Strength and agility training in adolescents with Down syndrome: A randomized controlled trial. Research in Developmental Disabilities, 33, 2236-2244.

[24] Jankowicz-Szymanska, A., Mikolajczyk, E., Wojtanowski, W. (2012). The effect of physical training on static balance in young people with intellectual disability. Research in Developmental Disabilities, 33, 675-681. doi.org/10. 1016/j.ridd. 2011. 11. 015.

[25] Aizawa, C., Morales, M., Lundberg, C., Soares de Moura, M., Pinto, F., Voos, M., \& Hasue, R. ( 2017). Conventional physical therapy and physical therapy based on reflex stimulation showed similar results in children with myelomeningocele. Arq. Neuro-Psiquiatr. 75(3), 160-166.

[26] Wuang, T., \& Tsai, H. (2017). Sensorimotor and visual perceptual functioning in school-aged children with Williams syndrome. Journal of Intellectual Disability Research, 61(4), 348-362.

[27] Hosseine, S., Ghoochani, B., Talebian, S., Pishyare, E., Haghgoo, H., Meymand, R., \& Zeinalzadeh, A. (2015). Investigating the effects of vestibular stimulation on balance performance in children with cerebral palsy: A randomized clinical trial study. Journal of Rehabilitation Sciences and Research, 2, 41-46. 